Human Ethology Bulletin 31 (2016)4: 1- 4

Letter from the Editorial Board

\title{
COMPROMISES - THE LIMITS OF DEMOCRACY
}

\section{Elisabeth Oberzaucher}

\section{Editor in Chief}

How do groups find consensus in decisions? In group-living species like Homo sapiens there are two basic possibilities how decisions are being made about the group's behaviour: Either there is a more or less democratic process of decision making, or future actions are decided upon hierarchically by a leader. If decision making is the responsibility and the privilege of one animal, the decisions can be made in a fast manner, at the cost of not always leading to solutions that distribute costs and benefits evenly. Democratic processes take longer, but promise to lead to fairer solutions.

Ideally, all group members pursue goals that are in agreement and can therefore work together to achieve them. It rarely happens that individual goals are in complete harmony, which is why an absence of conflicts is unrealistic in any group.

The ideas and wishes of individuals are not necessarily compatible. The greatest desire of one can be the worst nightmare of the other. The farther apart the goals of the group members, the larger are the difficulties that can make a resolution difficult. If consolation of conflicting interests cannot be achieved, there are two possible outcomes: Either an escalation of the conflict is prevented through avoidance behaviour, or, if that is not possible, the disagreement can lead to open conflict. There is a third option that lies between those extremes: A compromise is found by both parties giving up their initial goals partially, thus enabling both to achieve parts of what they desire.

Physical conflicts are disadvantageous for everyone involved. Therefore there is usually a tendency to avoid escalation of conflicts to the point when it results in a physical fight. Physical conflict serves as a last resort when all other 
means have failed to resolve the issue. The massive costs caused by physical aggression make compromises a much more attractive option. A compromise means that one does not achieve one's goal to the whole extend, but does so at least partially. Additionally, the massive costs of open conflict (injuries and loss of material benefits) can be avoided. (Savin-Williams, 1979; Yamamura \& Higashi ,1992)

Finding a compromise can be time consuming and difficult, but social structures can help in facilitating such processes: Implicit rules help us to reduce conflicts by regulating social interactions. Hierarchies and territoriality exist primarily in order to provide a framework of social rules to modulate interactions and reduce conflict. Both, hierarchies and territoriality, have a bad reputation in our every-day language, and are not necessarily associated with harmonising social groups. This is mostly due to the misrepresentation of aggression related to hierarchy and territory in documentaries, where they usually are shown in great detail and high frequency. In real life social interactions fights for social ranks or territories are very rare. They happen only when subordinate individuals fail to recognise the claim of the dominant individual or the owner of the territory. (Hamilton, 1971)

Both hierarchies and territories are respected because not only the individuals who have high status or own a territory benefit from it, but the subordinates do so, too. They might not do so to the same degree, but the benefits of playing along with the implicit rules outweigh the costs of a conflict. Both mechanisms regulate the rights and duties of the group members and reduce thus the likelihood of escalating conflicts. High ranking individuals do gain a higher benefit, but the low ranking individuals gain by reduced aggression. Territories are the basis for dominance linked to space and therefore have a similar function as hierarchies. The owner of a territory can set the rules, and they will be respected by the others most of the time. In case the demands of the high ranking individual or the owner of a territory are outrageous, the other group members might challenge their claims. If the claims appear to be unfounded for the others, they might challenge them in an open conflict. (Brown \& Orians, 1970)

Fairness plays a central role whether a compromise works out or not. If we feel that we have been treated unfairly, we rise to stand up for our own interest. There seems to be an innate understanding for what is acceptable in group living species. Nonhuman primates respond to being treated unfairly with angry responses (Brosnan, 2013). Recent studies have shown similar behaviours in dogs, too: They seem to care about the value of a reward only, if others receive a better reward than themselves. If that happens, they respond first 
with angry refusal of the reward, and then with a refusal to show the behaviour that was rewarded in the first place. (Range, Horn, Viranyi \& Huber, 2009)

When we think about fairness it seems to be less important whether our own needs are satisfied, but we seem to focus on what others get. This sense for injustice comes with the dark face of envy - social comparison makes it hard for us not to begrudge others what they have. The adaptive value is that it enables us to monitor fairness in a social system. Through social comparison we ensure that compromises are not made at the cost of a few, but in a equally distributed manner. A good compromise ensures that all involved parties invest and benefit to a comparable degree. This mechanism works quite well in small groups where individuals who violate the rules can be easily identified, and thus be kept from benefiting at the cost to all others. Such free riders can either be excluded from the mutual support system or be actively punished for their violation of reciprocal rules. In today's anonymous societies this mechanism is not suitable to execute this kind of control, what remains is the feeling of envy. Our evolved strategies do not suffice to deal with the social complexity of modern societies, and therefore the compromises found in democratic processes are not always fair ones. The dissociation of responsibility and power leads to a lack of individual responsibility, that makes this political system vulnerable to exploitation through egoistic and short term profit oriented people. (Festinger, 1954; Trivers, 1971; Axelrod \& Hamilton, 1981)

\section{Dissonance as opportunity}

If harmony is omnipresent and there is no disagreement, there is no creativity. New ideas usually originate from someone who does differently from everyone else, who tries new approaches and comes up with novel solutions. A perfectly harmonious world therefore might promise to be peaceful, but is likely unrealistic as it would fail to foster innovation. Inertia is dangerous on the biological and on the cultural level. Innovations in the sense of genetic mutations are the basic precondition for evolution that lead to the development of new characteristics, or even new species depending on the selection pressures that demand a deviation from the established ways. Since unpredictable changes are an integral part of our world, we have to remain flexible in our responses. The survival of any system - biological or social depends on its ability to maintain flexibility through continuous variation and innovation. The misfits, the revolutionaries are the ones that keep a society on its toes, and demand it to stay awake and innovative. A certain amount of conflict or disagreement facilitates cultural development, as this fosters creativity and innovation. A society will have the best prospect of succeeding on the long term, if it manages to maintain a culture of disagreement as a 
spawn for creativity, while keeping disagreements escalating into open conflict. (Mayr 1963)

\section{Power and Responsibility}

In group living species with hierarchical structures, a high social rank is usually associated with more responsibility and higher risks. The dominant animal benefits from its elevated status through privileges, such as priority access to resources, but is also responsible for the wellbeing of the group. For example, the alpha male is expected to face danger upfront. If the responsibility is not taken up, a loss of rank is the immediate consequence. This immediate connection between social rank and responsibility remained in place up until the medieval ages: Social contracts bound the liege and the vassal equally. If either failed to keep his part, the other was entitled to challenge the mutual dependency. This correlation is dissolved in modern democracies: A failure to keep the bargain does not necessarily lead to a loss of status. Direct control of fairness in compromises is further hindered by the scale on which they are found. The complexity of most processes makes it difficult to determine who is responsible. (DeCelles, DeRue, Margolis, \& Ceranic, 2012)

We employ democratic processes in order to find solutions that are fair for everyone. Control is executed in regular elections - a compromise that ensures that democracy remains the best form of government.

The complexity of modern societies poses a novel challenge to us for which we have no suitable evolved solution. The mechanisms that worked well in smaller groups are not so great at dealing with the demands brought about by the new scale and complexity. This is why new solutions have to be found on the intersection of nature and culture.

\section{REFERENCES}

Axelrod, R. \& Hamilton, W. D. The evolution of cooperation. Science 211, 1390 (1981). DOI

Brosnan, S. F. (2013). Justice- and fairness-related behaviors in nonhuman primates. Proceedings of the National Academy of Sciences of the United States of America, 110(Suppl 2), 10416-10423. DOI

Brown, J. L. \& Orians, G. H. 1970. Spacing patterns in mobile animals. - Ann. Rev. Ecol. 2: 239-262. DOI 
DeCelles, K. A., DeRue, D. S., Margolis, J. D. \& Ceranic, T. L. (2012). Does power corrupt or enable? When and why power facilitates self-interested behavior. Journal of Applied Psychology, 97, 681-689. DOI

Festinger L (1954). A theory of social comparison processes. Human relations. 7 (2): 117-140. DOI

Hamilton, W.D. (1971). "Geometry for the Selfish Herd". Journal of Theoretical Biology. 31 (2): 295-311. DOI

Mayr, Ernst (1963). Animal Species and Evolution. Cambridge: Harvard University Press. DOI

Range, F., Horn, L., Viranyi, Z., Huber, L. (2009) The absence of reward induces inequity aversion in dogs. PNAS 2009106 (1) 340-345. DOI

Savin-Williams, R. (1979). Dominance Hierarchies in Groups of Early Adolescents. Child Development, 50(4), 923-935. DOI

Trivers, R. (1971). The evolution of reciprocal altruism. Q. Rev. Biol. 46, 3557. DOI

Yamamura, N. \& M. Higashi (1992) An evolutionary theory of conflict resolution between relatives: altruism, manipulation, compromise. Evolution 46: 1236-1239. DOI 